\title{
Subject Index Vol. 34, 1996
}

\section{Addiction 136}

Add-on therapy 204

Adrenaline 82

Affective disorders 22

Age 201

Aging 18

Alprazolam 36

Antidementia drug 29

Antidepressants 26,98

Antidepressive medication 204

Antihypoxidotics 29

Anxiety 208

Augmenting and reducing 130

Autism 72

Autoimmune disorders 72

Automated drug classification 44

Befloxatone 98 Benzodiazepine(s) 9, 49, 90 binding inhibitory activity 9

Biochemical and psychological responses

36

- $\quad$ indicators 146

Blood platelets 184

Brain dialysis 82

C4Bgene 72

Calcium 26

Carbamazepine 22

Central benzodiazepine receptors 9

Cerebral blood flow 175

CGS19281A 82

Challenge tests 155

Childhood behavior 146

Chronic fatigue syndrome 175

Correlates 192

Corticosterone 136

Cortisol 155

Criminal offence 146

Dementia of Alzheimer type 29 Depression 18, 175

spectrum disease 188 Dichotic listening 57 Dimensions 192 Dopamine 155

agonists 117 Dopaminergic neurons 136 Dosage, multiple 113 -, single 113

EEG 106,208 Electroconvulsive therapy 204 
Electrocorticogram 44 Electroencephalography 98 Endogenous ligands 9 ß-Endorphin 18 Event-related potential(s) 49, 57, 98 Exploration 136

Fatigue 175

Food reinforcement 136

Gender 113 GH 82

Healthy conditions 9

- $\quad$ male persons 201

Heart rate 208

Histology 117

Hydrocephalus 76

E-10-Hydroxynortriptyline 44

Hypodopaminergia 76

Immunology 63

Inhibition 57

Intracellular $\mathrm{Ca} 2+$ mobilization 22

Ischaemia 117

Kidney 113

Linear prediction 1 Lisuride 117 Lithium 113 Longitudinal research 146

Magnesium 26 Major depression 204

depressive disorder 26

histocompatibility complex $72 \mathrm{MAO}$ inhibitors 98

Matched filtering 166 Memory-guided saccades 67 MHPG 82, 188 Mood 90

- $\quad$ disorders 188

Morris water maze 117

Multi-infarct dementia 29

Neuroactive steroid 106 Neurophysiology 192 Neuropsychology 14, 175, 192 Nicotine 208

deprivation 155

yield 208 Nootropics 29

223

Norm-breaking 146 Nullallele 72

Obsessive-compulsive disorder 63, 184 Ocular-motor delayed-response task 67

P300 57

$3 / 4$-Paroxetine 184

Peripheral blood mononuclear cells 18

Personality scales 146

Pharmacodynamics 98

Pharmaco-EEG 98

Phenobarbital 90

Phosphatidylserine 18

Piribedil 117

Placebo-controlled phase III trials 29

Platelet 5-HT concentrations 201

- monoamine oxidase activity 146

PNMT inhibitors 82

Prefrontal cortex 14 Pregnanolone 106 Premedication 90 Prolactin 155 Promethazine 90 Psychiatric disorders 9 Psychological stress 36

Rat C6BU-1 glioma cells 22

Recognition memory 49

Recurrent (unipolar) major depression 201

REM-NREM sleep cycle 1

Roman high avoidance rats 130

- $\quad$ low avoidance rats 130 
Schizophrenia 14, 57, 67, 192, 201 Schizophreniform catatonia 76 Sedation 106 Selective attention 57 Self-report questionnaire 90 Sensation seeking 130,155 Serotonin 22,72,155, 184

transporter 184 Signal detection theory 166

processing 166 SKF64139 82 Sleep EEG 1

Slow wave activity 1

Smoking 208

Sodium valproate 22

SPECTscan 175

Spectral analysis 1

Stimulus detectability 166

Subthalamic mesencephalic tumor 76

$\mathrm{T}$ cells 63

Transcranial magnetic stimulation 204

Treatment 188

Twin studies 14

14-UnitT-maze 117

4-Vessel occlusion 117 Vigilance control 44 Vincamine 29 Visual drug classification 44 evoked potentials) 130,166

search 49

Wisconsin Card Sorting Test 14 Zolpidem 90

224 
